\title{
Size Effects on the Electrical Resistivity of the Ultra-Thin Metallic Film
}

\author{
A. PAJA* AND A. DZIAlO \\ AGH University of Science and Technology, Faculty of Physics and Applied Computer Science \\ Department of Solid State Physics, al. A. Mickiewicza 30, 30-059 Krakow, Poland
}

(Received November 29, 2013)

\begin{abstract}
In this article we investigate the electron-phonon interaction in metals in the system strongly reduced in one dimension. The Fermi sphere which represents the free-electron structure of a bulk metal was replaced by a discrete set of the Fermi disks. Using the variational expression for resistivity the temperature and film thickness dependences of the resistivity were derived and compared with experimental data.
\end{abstract}

DOI: 10.12693/APhysPolA.125.1220

PACS: 73.50.-h, 73.50.Bk, 73.63.-b, 73.63.Hs

\section{Introduction}

It is undoubtedly that the properties of bulk materials and materials nanoscaled at least in one dimension are different. The behaviour of electrons in systems having dimensions of the intermediate length scale has been actively studied in past few decades. Naturally the main target of investigation and research were semiconductors, so much important in electronics, optoelectronics, quantum electronics etc. In semiconductors the carrier scattering at room temperatures is dominated by the polar-optical-phonon scattering mechanism. Scattering rates of carriers by longitudinal optical phonons in semiconducting quantum well structures are greater than those in the same bulk materials [1]. First studies of quantum size effects in thin semimetallic films with perfect surfaces were performed by Sandomirskii [2]. He showed that the electrical conductivity, the Hall constant, and magnetoresistance had an oscillating dependence as the functions of the thickness of the film. More extended work by Ashcroft and Trivedi [3] involved discreteness of the energy levels, effects of impurity potentials, and surface roughness. Our goal is to find the temperature effect on the electrical resistivity of a very thin metallic layer.

We previously derived the formula for exactly two-dimensional metallic system [4]. We showed that at low temperatures the electrical resistivity of bulk material was higher than that of the two-dimensional system and this effect was purely geometrical. The main disadvantage of this model is that it is strictly two-dimensional and can only be treated as a "toy" model. That is why this time we investigated more realistic quasi two-dimensional system representing a very thin metallic layer. Of course we used the free-electron model which we consider to be simple and easy to handle yet it allows us to describe basic properties of a metal.

\footnotetext{
*corresponding author; e-mail: paja@agh.edu.pl
}

\section{Theoretical model}

For our calculations of the film resistivity we take a simple particle-in-a-box model in which independent electrons are confined by an infinitely deep and smooth (perfect surfaces) potential. The phonons are taken to be bulk ones. The eigenvalue spectrum and eigenfunctions for such quantum well are given by

$$
\begin{aligned}
& \varepsilon_{k, \tau}=\frac{\hbar^{2} k^{2}}{2 m^{*}}+\varepsilon_{0} \tau^{2}, \\
& \psi_{k, \tau}(r)=\sqrt{\frac{2}{V}} \sin \left(\frac{\pi \tau}{L_{z}} z\right) \mathrm{e}^{\mathrm{i} \boldsymbol{k} \cdot \boldsymbol{\rho}},
\end{aligned}
$$

where $\boldsymbol{k}$ is a continuous, in-plane wave vector, $\tau$ is an integer index of a specific subband, $\varepsilon_{0}=\hbar^{2} \pi^{2} / 2 m^{*} L_{z}^{2}$ is the ground state energy, $\rho$ is a vector in the $x-y$ plane and $V=A L_{z}$ is the volume of the film.

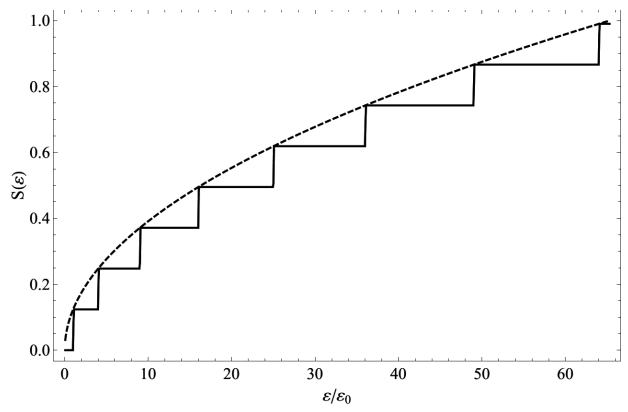

Fig. 1. Exemplary density of states $S(\varepsilon)$ in arbitrary units as a function of $\varepsilon / \varepsilon_{0}$ for a system with the thickness of 5 atomic layers.

For the eigenvalues given above the density of states per unit energy interval per unit volume (without taking account of the spin degeneracy) is

$$
S(\varepsilon)=\frac{1}{4 \pi^{2}} \sqrt{\frac{2 m^{*} \varepsilon_{0}}{\hbar^{2}}} \operatorname{Int}\left(\sqrt{\varepsilon / \varepsilon_{0}}\right),
$$

where Int denotes the integer part of the argument. The density of states function is a step function with an en- 


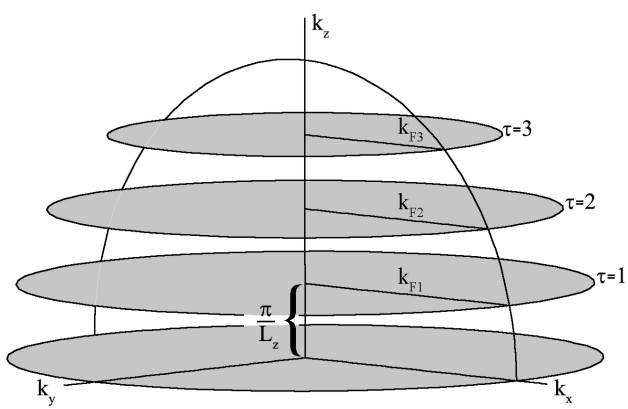

Fig. 2. In a thin layer the Fermi sphere reduces to a set of parallel disks separated by the distance $\pi / L_{z}$. Electrons can occupy only those states included within the disk.

velope described by three-dimensional density of states (Fig. 1).

Consistently the Fermi sphere is reduced to a set of flat Fermi surfaces [5-7] where electronic states are contained within the circles of the radii $k_{\mathrm{F} \tau}(\tau=1,2,3 \ldots)$. These circles are the cross-sections of the sphere of the radius $k_{\mathrm{F}}$ and the planes perpendicular to the $z$-axis, separated by the distances $\pi / L_{z}$ (Fig. 2). The concentration of electrons is given by

$$
n_{\mathrm{e}}=\frac{\tau_{\mathrm{F}}}{2 \pi L_{z}}\left[k_{\mathrm{F}}^{2}-\frac{\pi^{2}}{6 L_{z}^{2}}\left(\tau_{\mathrm{F}}+1\right)\left(2 \tau_{\mathrm{F}}+1\right)\right],
$$

which we assume to be constant, independent of film thickness and equal to the bulk one, hence the Fermi wave vector must vary to contain all the available electrons. Here $\tau_{\mathrm{F}}$ is the maximal natural number, for which the intersection of the plane $k_{z}=\tau \pi / L_{z}$ with the sphere with radius $k_{\mathrm{F}}$ is still possible.

\section{Size and temperature dependent resistivity}

We assume that the variational expression [8] for the resistivity is still valid within our model

$$
\rho_{\mathrm{L}}=\frac{\frac{1}{k_{\mathrm{B}} T} \iiint\left(\phi_{\boldsymbol{k}}-\phi_{\boldsymbol{k}^{\prime}}\right)^{2} \mathcal{P}_{\boldsymbol{k}, \boldsymbol{q}}^{\boldsymbol{k}^{\prime}} \mathrm{d} \boldsymbol{k} \mathrm{d} \boldsymbol{k}^{\prime} \mathrm{d} \boldsymbol{q}}{\left|\frac{1}{4 \pi^{3}} \int e \boldsymbol{v}_{\boldsymbol{k}} \cdot \boldsymbol{u} \phi_{\boldsymbol{k}} \frac{\partial f_{\boldsymbol{k}}^{0}}{\partial \boldsymbol{\varepsilon}_{\boldsymbol{k}}} \mathrm{d} \boldsymbol{k}\right|^{2}},
$$

where $\boldsymbol{k}, \boldsymbol{k}^{\prime}$ - electron wave vectors before and after scattering, respectively, $\boldsymbol{q}$ - phonon wave vector, $k_{\mathrm{B}}$ the Boltzmann constant, $T-$ temperature, $\phi_{k}-$ deviation from equilibrium of the electron distribution function, $\mathcal{P}_{\boldsymbol{k}, \boldsymbol{q}}^{\boldsymbol{k}^{\prime}}$ - transition probability from the state $\boldsymbol{k}$ to the state $\boldsymbol{k}^{\prime}$ due to electron-phonon interaction, $e-$ electron charge, $\boldsymbol{v}_{\boldsymbol{k}}$ - electron group velocity, $f_{k}^{0}$ - electron equilibrium distribution function, $\varepsilon_{k}$ - electron energy, $\boldsymbol{u}$ - unit vector in the direction of external field.

The choice of trial function is the matter of the scattering problem we would like to solve. Various trial functions are used in cases of elastic scattering on impurities, inelastic scattering on phonons and occurrence of thermal gradient $[9,10]$. We assume thermal equilibrium, no thermal gradient and only phonon-electron interactions with no impurities in the system thus we choose the trial function to be $[8,11]$ :

$$
\phi_{\boldsymbol{k}}=\boldsymbol{k} \cdot \boldsymbol{u} \text {. }
$$

Performing simple calculations we see that the denominator is then given by

$$
\left|\frac{1}{4 \pi^{3}} \int e \boldsymbol{v}_{\boldsymbol{k}} \cdot \boldsymbol{u} \phi_{\boldsymbol{k}} \frac{\partial f_{\boldsymbol{k}}^{0}}{\partial \varepsilon_{\boldsymbol{k}}} \mathrm{d}^{3} k\right|^{2}=\left(\frac{e n_{\mathrm{e}}}{\hbar d}\right)^{2},
$$

where $d$ is a constant depending on the system symmetry.

The numerator in Eq. (4) is much more complex. First we shall take care of the transition probability due to electron-phonon interactions employing the standard formula of the Fermi golden rule

$$
\mathcal{P}_{\boldsymbol{k}, \boldsymbol{q}}^{\boldsymbol{k}^{\prime}}=\frac{2 \pi}{\hbar}\left|M\left(k, k^{\prime}\right)\right|^{2} \mathcal{O}\left(\varepsilon_{\boldsymbol{k}}+\hbar \omega-\varepsilon_{\boldsymbol{k}^{\prime}}\right) n_{\boldsymbol{q}}^{0} f_{\boldsymbol{k}}^{0}\left(1-f_{\boldsymbol{k}^{\prime}}^{0}\right),
$$

where $n_{q}^{0}$ is the Bose-Einstein distribution function.

The matrix element for a particular lattice mode of wave vector $\boldsymbol{q}$ and polarisation $p$ takes the form

$$
\begin{aligned}
& M\left(k, k^{\prime}\right)=\left\langle n_{q, p}\left|\int \psi_{k}^{*}(r) \mathcal{H}_{e p} \psi_{k^{\prime}}(r) \mathrm{d} r\right| n_{q, p}-1\right\rangle \\
& =\sqrt{\frac{\hbar n_{q, p}}{2 m_{i} N V \omega_{q}}} \\
& \quad \times \sum_{l} \mathrm{e}^{-\mathrm{i} q l} \int \psi_{k}^{*}\left(\boldsymbol{e}_{q, p} \cdot \nabla U(r)\right) \psi_{k^{\prime}} \mathrm{d} r,
\end{aligned}
$$

where $m_{i}$ - ion mass, $N$ - number of unit cells per volume. If the potential $U(r)$ can be separated into two parts, namely the in-plane $U(x, y)$ and perpendicular-to-plane $U(z)$ :

$$
U(\boldsymbol{r})=U(x, y)+U(z),
$$

then the integration in the matrix element expression can be simplified as follows:

$$
\begin{aligned}
& \int \psi_{k}^{*}(r)\left(e_{q, p} \cdot \nabla U(r)\right) \psi_{k^{\prime}}(r) \mathrm{d} r \\
& =\int \psi_{k}^{*}(r)\left(e_{x, y} \cdot \nabla U(x, y)\right) \psi_{k^{\prime}}(r) \mathrm{d} r,
\end{aligned}
$$

since potential $U(z)$ corresponds to the geometrical confinement and is constant along the $z$ axis. After substituting the second of Eqs. (1) into Eq. (8), the matrix element takes the form

$$
M\left(k, k^{\prime}\right)=\sqrt{\frac{\hbar n_{q, p}}{2 m_{i} N V \omega_{q}}} \delta_{g, k_{\|}^{\prime}-k_{\|}-q} \mathcal{I}_{q, p}\left(k_{\|}, k_{\|}^{\prime}\right),
$$

with the factor

$$
\mathcal{I}_{q, p}\left(k_{\|}, k_{\|}^{\prime}\right)=\int \varphi_{k}^{*}\left(e_{x, y} \cdot \nabla U(x, y)\right) \varphi_{k^{\prime}} \mathrm{d} x \mathrm{~d} y
$$

to be determined.

Now the final expression for the transition probability is given by

$$
\begin{aligned}
& \mathcal{P}_{k, q}^{k^{\prime}}=\frac{2 \pi}{\hbar}\left(\frac{\hbar}{2 m_{i} N V \omega_{q}}\right) \delta_{g, k_{\|}^{\prime}-k_{\|}-q}\left|\mathcal{I}_{q, p}\left(k_{\|}, k_{\|}^{\prime}\right)\right|^{2} \\
& \quad \times \mathcal{O}\left(\varepsilon_{k}+\hbar \omega-\varepsilon_{k^{\prime}}\right) n_{q}^{0} f_{k}^{0}\left(1-f_{k^{\prime}}^{0}\right) .
\end{aligned}
$$

Various mechanisms of electron-phonon scattering give their contributions to the function $\mathcal{I}_{q, p}\left(k, k^{\prime}\right)$, which can be derived in many various ways. In every case we find 
that for longitudinal phonons

$$
\mathcal{I}_{q, p}\left(k_{\|}, k_{\|}^{\prime}\right)=\boldsymbol{e}_{q, p} \cdot\left(\boldsymbol{k}_{\|}^{\prime}-\boldsymbol{k}_{\|}\right) \mathcal{C}\left(\left|\boldsymbol{k}_{\|}^{\prime}-\boldsymbol{k}_{\|}\right|\right),
$$

where $\mathcal{C}\left(\left|\boldsymbol{k}_{\|}^{\prime}-\boldsymbol{k}_{\|}\right|\right)$is a quantity which has the dimension of energy and depends on the magnitude of the scattering vector

$$
K_{\|}=\left|\boldsymbol{k}_{\|}^{\prime}-\boldsymbol{k}_{\|}\right| \text {. }
$$

Thus the integral in the numerator of the variational expression for the electrical resistivity is

$$
\begin{aligned}
& \frac{\pi}{k_{\mathrm{B}} T} \iiint \frac{\left(\boldsymbol{K}_{\|} \cdot \boldsymbol{u}\right)^{2}\left(\boldsymbol{K}_{\|} \cdot \boldsymbol{e}\right)^{2} \mathcal{C}^{2}\left(K_{\|}\right)}{m N \omega_{q}} n_{q}^{0} f_{k}^{0}\left(1-f_{k^{\prime}}^{0}\right) \\
& \quad \times \mathcal{O}\left(\varepsilon_{k}+\hbar \omega-\varepsilon_{k^{\prime}}\right) \delta_{g, k_{\|}^{\prime}-k_{\|}-q} \mathrm{~d}^{3} k \mathrm{~d}^{3} k^{\prime} \mathrm{d}^{3} q .
\end{aligned}
$$

First we deal with the energy conservation condition, by integrating over the magnitude of $\mathrm{d} \boldsymbol{k}^{\prime}$ :

$$
\begin{aligned}
& \int \mathcal{O}\left(\varepsilon_{k}+\hbar \omega-\varepsilon_{k^{\prime}}\right) \mathrm{d}^{3} k^{\prime} \\
& =\frac{\pi}{L_{z}} \frac{1}{8 \pi^{3}} \sum_{k_{z}^{\prime}} \iint \mathcal{O}\left(\varepsilon_{k}+\hbar \omega-\varepsilon_{k^{\prime}}\right) \frac{\mathrm{d} \varepsilon_{k^{\prime}}}{\hbar v_{k^{\prime}}} \mathrm{d} \varsigma^{\prime} \\
& =\frac{\pi}{L_{z}} \frac{1}{8 \pi^{3} \hbar} \sum_{k_{z}^{\prime}} \int \frac{\mathrm{d} \varsigma^{\prime}}{v_{k^{\prime}}} .
\end{aligned}
$$

In this transformation we drop the term coming from the rate of change of $\hbar \omega$ with $\boldsymbol{k}^{\prime}$. This is small, in comparison with the velocity of sound, and will be neglected. Now we are left with only one integral over $\mathrm{d} \boldsymbol{k}$ and the integrand

$$
\begin{aligned}
& \int f_{k}^{0}\left(1-f_{k^{\prime}}^{0}\right) \mathrm{d}^{3} k=\frac{\pi}{L_{z}} \frac{1}{4 \pi^{3} \hbar} \sum_{k_{z}} \int \frac{\mathrm{d} \varsigma}{v_{k}} \\
& \times \int \frac{\mathrm{d} \varepsilon_{k}}{\left(\mathrm{e}^{\left(\varepsilon_{k}-\mu\right) / k_{\mathrm{B}} T}+1\right)\left(\mathrm{e}^{-\left(\varepsilon_{k}+\hbar \omega-\mu\right) / k_{\mathrm{B}} T}+1\right)} \\
& =\frac{\pi}{L_{z}} \frac{k_{\mathrm{B}} T}{4 \pi^{3} \hbar} \sum_{k_{z}} \int \frac{\mathrm{d} \varsigma}{v_{k}} \int \frac{\mathrm{d} w}{\left(\mathrm{e}^{w}+1\right)\left(\mathrm{e}^{-(w+t)}+1\right)} \\
& =\frac{\pi}{L_{z}} \frac{k_{\mathrm{B}} T}{4 \pi^{3} \hbar} \frac{t}{1-\mathrm{e}^{-t}} \sum_{k_{z}} \int \frac{\mathrm{d} \varsigma}{v_{k}},
\end{aligned}
$$

where $w=\left(\varepsilon_{k}-\mu\right) / k_{\mathrm{B}} T$ and $t=\hbar \omega / k_{\mathrm{B}} T$.

Putting all the partial results into the integration together and integrating over $\varsigma^{\prime}$ we obtain

$$
\begin{aligned}
\rho_{\mathrm{L}} & =\frac{\hbar d^{2}}{64 \pi^{3} k_{\mathrm{B}} T e^{2} n_{\mathrm{e}}^{2} m N v_{\mathrm{F}}^{2}} \sum_{\tau^{\prime}} \varsigma_{\tau^{\prime}} \\
& \times \sum_{k_{z}} \int \frac{(\boldsymbol{K} \cdot \boldsymbol{u})^{2}\left(\boldsymbol{K}_{\|} \cdot \boldsymbol{e}\right)^{2} \mathcal{C}^{2}\left(K_{\|}\right) k_{\mathrm{F} \tau} \mathrm{d} \phi}{\left(1-\mathrm{e}^{-\hbar \omega_{q} / k_{\mathrm{B}} T}\right)\left(\mathrm{e}^{\hbar \omega_{q} / k_{\mathrm{B}} T}-1\right)},
\end{aligned}
$$

where $\phi$ is the in-plane scattering angle. For $N$-processes mainly the longitudinal lattice waves cause the scattering, thus vector $\boldsymbol{e}$ is parallel to $\boldsymbol{q}$ which equals $\boldsymbol{K}$. That is why we take their scalar product to be

$$
\left(\boldsymbol{K}_{\|} \cdot \boldsymbol{e}\right)^{2}=q^{2} \text {. }
$$

We stated at the beginning that the thin metallic layer is in contact with the non-conducting basis. For this reason the phonon spectrum in the layer is the same as the bulk phonon one. Since $\left(\boldsymbol{K}_{\|} \cdot \boldsymbol{u}\right)^{2}$ depends on the system symmetry we can replace it by $(1 / d) K^{2}$. Because only $N$ processes take place, thus the $\mathcal{C}^{2}$ in the numerator in Eq. (19) is independent of $q$ and is equal to (using deformation potential approximation):

$$
\mathcal{C}^{2}\left(q_{\|}\right)=\mathcal{C}^{2}(0)=\left(\frac{n_{\mathrm{e}}}{S\left(\varepsilon_{\mathrm{F}}\right)}\right)^{2},
$$

where $S\left(\varepsilon_{\mathrm{F}}\right)$ is the density of states at the Fermi level given by Eq. (2). To accomplish the calculations, all we need is to find $k_{\mathrm{F} \tau} \mathrm{d} \phi$ expressed by the scattering vector $q$. This can be done by using the law of cosines in the form

$$
\begin{aligned}
& q^{2}=k^{\prime 2}+k^{2}-2 k^{\prime} k \cos \phi \Longrightarrow q \mathrm{~d} q=k_{\mathrm{F} \tau}^{2} \sin \phi \mathrm{d} \phi, \\
& q^{2}=k^{\prime 2}+k^{2}-2 k^{\prime} k \cos \phi \Longrightarrow \cos \phi=1-\frac{q^{2}}{2 k_{\mathrm{F} \tau}^{2}} .
\end{aligned}
$$

Finally the equation for the electrical resistivity of such quasi-two-dimensional metallic layer is

$$
\begin{gathered}
\rho_{\mathrm{L}}=\frac{\hbar d}{64 \pi^{3} k_{\mathrm{B}} T e^{2} m N v_{\mathrm{F}}^{2} S_{\varepsilon_{\mathrm{F}}}^{2}} \sum_{\tau^{\prime}} \varsigma_{\tau^{\prime}} \sum_{k_{z}} \\
\times \int \frac{q^{4} \mathrm{~d} q}{\left(1-\mathrm{e}^{-\hbar \omega_{q} / k_{\mathrm{B}} T}\right)\left(\mathrm{e}^{\hbar \omega_{q} / k_{\mathrm{B}} T}-1\right) \sqrt{1-\frac{q^{2} L_{z}^{2}}{4\left(k_{\mathrm{F}}^{2} L_{z}^{2}-\tau^{2} \pi^{2}\right)}}} .
\end{gathered}
$$

\section{Results}

We calculated the electrical resistivity for copper films with given thickness in a range of temperature from $0 \mathrm{~K}$ up to $1.5 \theta$ where $\theta$ is the Debye temperature ( $c a .320 \mathrm{~K}$ for copper). The results of these calculations are shown in Fig. 3. The behaviour of the resistivity differs slightly from that of bulk metal but seems to be linear at high temperatures.

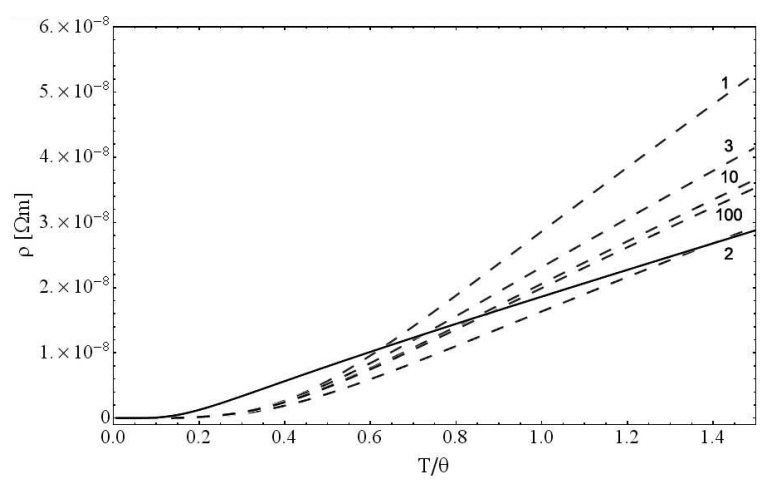

Fig. 3. Electrical resistivity of copper thin layers vs. reduced temperature for various film thicknesses (integers in the figure denote the number of the layers) and bulk copper resistivity (solid line).

We can see in Fig. 4 that the resistivity oscillates as the layer thickness changes. These oscillations quickly disappear and are negligible when $L_{z}$ equals to a few lattice constants. The cause of these oscillations is the varying density of states at the Fermi level.

What is important is that the behaviour of the resistivity shown in Fig. 4 weakly depends on the film thickness and at fixed temperature only oscillates around its bulk 


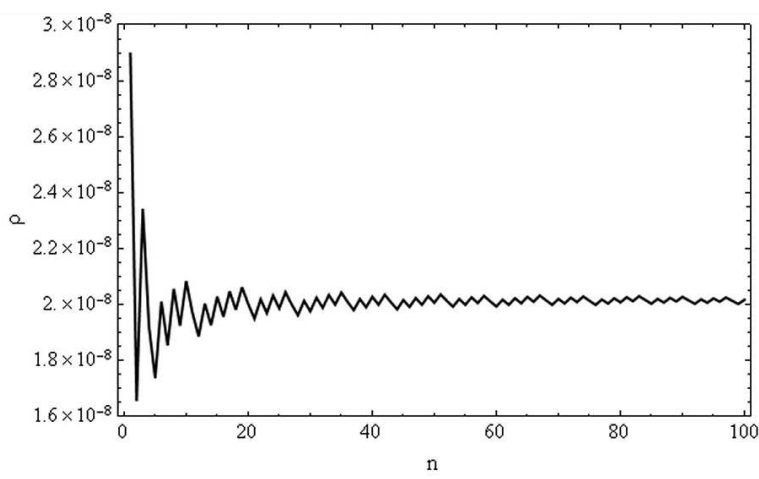

Fig. 4. Electrical resistivity $\rho$ for fixed temperature $(T=\theta)$ vs. number of monolayers $n$.

value. This result is in contrast with experimental data where the resistivity rises even several times as the thickness drops down and has a finite value for thickness of one atomic layer [12]. If our calculations are correct, then we suppose that the build-up in resistivity is brought on by surface roughness and cracks that may occasionally appear in the film.

\section{Conclusions}

In our opinion the obtained results are satisfying within the model. In our previous calculations [4] we observed rapid growth of resistivity up to infinity as temperature dropped down. Here this abnormal behaviour is not observed. More importantly in Fig. 4 we observe oscillations with layer thickness reported by various authors [3, 13] although these oscillations seem to behave in different way. In Fig. 3 we can see that resistivity for one hundred layers differs slightly from that obtained for bulk copper. We think that this is a result of assumption that scattering potential can be divided into two parts, the in-plane part and perpendicular-to-plane. That is why scattering takes place only in the plane and not in the direction of the $z$ axis. Additionally the square root expression appears in the denominator of final formula (23). Taking more realistic Coulomb-like pseudo-potential with screening and letting the scattering perpendicular to plane to happen could give us more satisfying results.

\section{Acknowledgments}

This work has been partly supported by the EU Human Capital Operation Program, Polish Project No. POKL.04.0101-00-434/08-00. It was also partially supported by the Polish Ministry of Science and Higher Education and its grants for Scientific Research.

\section{References}

[1] M.A. Stroscio, M. Dutta, Phonons in Nanostructures, Cambridge University Press, Cambridge 2005.
[2] V.B. Sandomirskii, J. Exp. Theor. Phys. 52, 158 (1967).

[3] N.W. Ashcroft, Nandini Trivedi, Phys. Rev. B 38, 17 (1988).

[4] A. Działo, A. Paja, Acta Phys. Pol. A 123, 770 (2013).

[5] B.J. Spisak, A. Paja, Mol. Phys. Rep. 40, 144 (2004).

[6] B.J. Spisak, A. Paja, Mater. Sci. - Poland 24, 605 (2006).

[7] T. Balcerzak, Thin Solid Films 500, 341 (2006).

[8] J.M Ziman, Electrons and Phonons, Clarendon Press, Oxford 1960.

[9] A.C. Ehrlich, Phys. Rev. B. 8, 3610 (1973).

[10] A.H. Wilson, Proc. Camb. Philos. Soc. 33, 371 (1937).

[11] F.J. Blatt, Physics of Electron Conduction in Solids, PWN, Warszawa 1973.

[12] G. Fischer, H. Hoffman, Solid State Commun. 35, 793 (1980).

[13] D.V. Fedorov, P. Zahn, I. Mertig, Thin Solid Films 473, 346 (2005). 